\title{
ResearchOnline@JCU
}

This is the Accepted Version of a paper published in the journal:

Journal of Sports Sciences

Guy, Joshua H., Edwards, Andrew M., Miller, Catherine M., Deakin, Glen B., and Pyne, David B. (2017) Short-term reliability of inflammatory mediators and response to exercise in the heat. Journal of Sports Sciences, 35 (16). pp. 16221628.

https://doi.org/10.1080/02640414.2016.1227464 


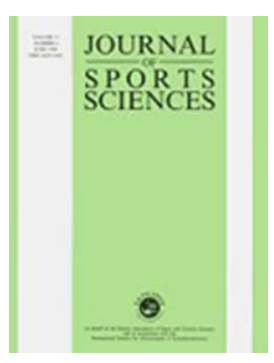

\section{Short-term reliability of inflammatory mediators in response to exercise in the heat}

\begin{tabular}{|r|l|}
\hline Journal: & Journal of Sports Sciences \\
\hline Manuscript ID & RJSP-2016-0124 \\
\hline Manuscript Type: & Original Manuscript \\
\hline Keywords: & $\begin{array}{l}\text { Lipopolysaccharide, heat shock proteins, inflammatory cytokines, heat } \\
\text { tolerance }\end{array}$ \\
\hline
\end{tabular}

\section{SCHOLARONE $^{\text {"n }}$ \\ Manuscripts}

URL: http://mc.manuscriptcentral.com/rjsp 
1 Manuscript Type: Original Investigation

2

3 Title: Short-term reliability of inflammatory mediators in response to exercise in the heat.

4 Running Title: Reliability of inflammatory mediators in response to exercise. 5

6

7

8 Word count:

9 Abstract: 194 words.

10 Manuscript: 3695 words. 


\begin{abstract}
11 Abstract
12 Prospective application of serum cytokines, lipopolysaccharide, and heat shock proteins

13 requires reliable measurement of these biomarkers of exercise-induced heat stress in hot

14 conditions. To accomplish this, both short-term (seven day) reliability (at rest, $\mathrm{n}=12$ ) and the

15 acute responsiveness of each biomarker to exercise in the heat (pre and post 60 min cycling,

$1634.5^{\circ} \mathrm{C}$ and $70 \% \mathrm{RH}, \mathrm{n}=20$ ) were evaluated. Venous blood was analysed for the serum

17 concentration of C-reactive protein (CRP), interleukin (IL)-6, heat shock protein 72

18 (eHSP72), immunoglobulin M (IgM) and lipopolysaccharide (LPS). Test-retest reliability

19 was determined as the coefficient of variation (CV). Biomarkers with the least short-term

20 within-subject variation were IL-6 $(19 \%, \pm 20 \% ; \mathrm{CV}, \pm 95 \%$ confidence limits $)$ and LPS

$21(23 \%, \pm 13 \%)$. Greater variability was observed for IgM, eHSP72 and CRP (CV range 28-

$2238 \%)$. IL-6 exhibited the largest increase in response to acute exercise $(95 \%, \pm 11 \%, p=$

$23<0.001)$ and although CRP had a modest CV $(12 \%, \pm 7 \%)$ it increased substantially post-

24 exercise $(p=0.02$, ES; 0.78). In contrast, eHSP72 and LPS exhibited trivial changes post-

25 exercise. It appears the mean change of common inflammatory markers after exercise in the

26 heat is not always discernible from short-term (weekly) variation.
\end{abstract}

27 Keywords Lipopolysaccharide, heat shock proteins, inflammatory cytokines, heat

28 tolerance. 


\section{Introduction}

30 Uncompensable heat stress experienced either passively or in response to exercise in the heat

31 influences a complex network of thermoregulatory, immune, inflammatory and

32 neuromuscular factors (Pyne, Guy, and Edwards, 2014). In extreme cases this inflammation

33 can culminate in multi-organ failure and even death (Singh, Kapoor, and Singh, 2013).

34 Induction of an inflammatory response plays an important role in this process after transient

35 heat can damage the gastrointestinal tract, causing it to become permeable, leading to leakage

36 of harmful bacterial endotoxins from the gut into the circulation. This effect is exacerbated by

37 exercise in the heat and, as a consequence, it is important to reliably evaluate the extent of

38 endotoxin leakage into circulation, and measure biochemical indices which accurately reflect

39 whether or not individuals are able to respond or adapt effectively to such exercise challenges

40 that occur in the heat.

42 Exercise-induced endotoxemia has been attributed primarily to lipopolysaccharide (LPS)

43 translocated from the gut into the circulation (Lim, et al., 2009). An abundance of circulating

44 LPS can evoke an inflammatory response, leading to heat shock and overwhelming of anti-

45 LPS mechanisms including the antibody, immunoglobulin M (IgM), (Cohen, Block, Green,

46 Lowell, and Ofek, 1989), and cytokines such as interleukin-6 (IL-6) operating in an anti-

47 inflammatory role (Abbasi et al., 2013). Therefore, when the anti-LPS mechanisms and rate

48 of LPS clearance are inadequate to counter the heat-induced increase of LPS, endotoxemia

49 may ensue.

51 A rise in extra cellular heat shock protein (eHSP) concentration is a consequence of an innate

52 immune response to whole body hyperthermia (Ahlers et al., 2005). In this scenario an acute

53 phase immune response is evoked to counteract heat-induced oxidative stress leading to an 
54 increase in leukocyte and eHSP concentrations (Mestre-Alfaro et al., 2012). Numerous

55 studies have demonstrated that non-critical exposure to heat may increase both tolerance to

56 oxidative stress and effectiveness of anti-LPS mechanisms (Pilch et al., 2014; Pyne et al.

57 2014; Yeh, Law, and Lim, 2013). Therefore, to accurately determine whether or not

58 individuals are susceptible to heat stress, or have effectively adapted over time to hot

59 conditions through heat acclimation or acclimatisation, it is important to quantify reliable,

60 relevant, and objective outcome measures of the immune and inflammatory responses.

61

62 Several studies have used blood biomarkers to quantify the magnitude of adaptation to hot

63 environmental conditions, although a comparison of short-term variability in heat-induced

64 biomarkers has not yet been conducted. This is surprising as there is considerable variation in

65 the magnitude of heat-induced change to markers such as interleukin (IL)-6, C-reactive

66 protein, LPS and eHSP72 following a bout of exercise in hot conditions (Hailes, Slivka,

67 Cuddy and Ruby 2011; Lim et al., 2009; Marshal, Campbell, Roberts, and Nimmo, 2007;

68 Rhind et al., 2004; Wright et al., 2013). As a common length for a short-term heat

69 acclimation protocol for athletes is seven days (Garrett, Rehrer, and Patterson, 2011) further

70 investigation into the variation of these biomarkers is warranted. The utility of individual

71 biomarkers may depend on typical variation (noise) under normal conditions, and the

72 magnitude of the response to exercise in the heat (signal). Another consideration is whether

73 the noise is sufficiently small so as to not mask biologically and/or clinically important

74 changes or differences. It is also plausible that while some biomarkers may demonstrate

75 considerable short-term variability, they could still be useful if the presentation of a heat

76 stimulus produces a sufficiently large signal (response). This is a point often overlooked in

77 the study of reliability of biomarkers. Consequently, evaluating reliability among heat-

78 relevant biomarkers should account for variation, and, importantly, whether the expected 
79 change in biomarker activity on presentation to a large heat and exercise stimulus is greater

80 than that of short-term variation.

81

82 The aim of this study was to quantify the reliability (short term test re-test reliability) in the

83 concentration of common inflammatory (blood) biomarkers at rest (twice over seven days,

84 Part A). A second aim was to examine the acute response of those biomarkers to an exercise

85 challenge performed in hot and humid conditions (Part B).

\section{Materials and methods}

88 Experimental Design

89

90 Part A: Short-term reliability of serum biomarkers.

91 This phase of the study was designed to examine the weekly variation in venous blood of

92 selected biomarkers in a non-exercise context and was conducted over 14 days (Figure 1).

93 The seven days preceding the first test day were used as a "lead-in" period and participants

94 were instructed to abstain from partaking in moderate -high intensity physical activity for the

95 duration of the study period. Participants then had venous blood drawn on two occasions

96 seven days apart. Blood was drawn approximately $2 \mathrm{~h}$ post-prandial at a similar time of day

97 (morning) to limit diurnal variation. At the beginning of the lead-in period all participants

98 undertook a baseline evaluation of maximum oxygen uptake $\left(\dot{V} \mathrm{O}_{2 \max }\right)$ using an incremental

99 treadmill running test to exhaustion. A seven day controlled lead-in or baseline period was

100 used to ensure that the participants were not suffering from any residual inflammatory effects

101 of exercise or illness prior to taking part in this study. Participants were instructed to maintain 
102 a similar dietary intake and (light) activity levels for $24 \mathrm{~h}$ preceding each venous blood

103 sample.

104 Part B: Acute response of serum biomarkers to exercise in the heat.

105 This phase of the study examined the acute response of biomarkers to exercise performed in

106 the heat. To aid robust evaluation of biomarkers free from influence of prior exercise, this

107 part of the study also contained a seven day lead-in period prior to assessment. At baseline,

108 all participants performed an incremental test to exhaustion for the assessment of $\dot{V} \mathrm{O}_{2 \max }$ on a

109 cycle ergometer - the same modality as the subsequent heat stress test protocol. As before, all

110 participants were required to abstain from moderate-high intensity exercise for the remainder

111 of the seven day lead-in period prior to further assessment of pre- to post-exercise evaluation

112 of biomarker activity. The exercise in the heat test occurred seven days after baseline

113 evaluation of $\dot{V} \mathrm{O}_{2 \max }$. Venous blood sampled for acute response to exercise in the heat was

114 drawn prior to and immediately following the heat stress test. Blood was sampled

115 approximately $2 \mathrm{~h}$ post-prandial at a similar time of day for all participants (morning) to limit 116 diurnal variation.

118 Subjects

119 Participants in Part A of this study (short-term variation) comprised twelve healthy

120 moderately-trained males (age $24.3 \pm 4.1$ years, $\dot{V} \mathrm{O}_{2 \max } 52.0 \pm 2.7 \mathrm{ml} . \mathrm{kg} \cdot \mathrm{min}^{-1}$, height

$121 \quad 1.78 \pm 0.09 \mathrm{~m}$, mass $73.9 \pm 8.5 \mathrm{~kg}$, mean $\pm \mathrm{SD}$ ). Part B participants (acute response to exercise

122 in the heat intervention) comprised twenty males (age 24.6 \pm 3.7 years, $\dot{V} \mathrm{O}_{2 \max } 43.2 \pm 5.4$

123 ml.kg. $\mathrm{min}^{-1}$, height $1.78 \pm 0.07 \mathrm{~m}$, mass $83.5 \pm 11.0 \mathrm{~kg}$ ). All participants completed a pre-

124 screening medical for use of immunomodulating medications (none were present). After

125 explanation of the study procedures, benefits and risks, participants provided written 
126 informed consent before inclusion in the project. This study was approved by the James Cook

127 University Human Research Ethics Committee and conformed to the guidelines set forth by

128 the Helsinki Declaration. Participants in Part A were also required to complete a daily

129 physical activity diary for the duration of the study so that any exercise undertaken could be

130 quantified for intensity and duration. All participants were also required to self-report any

131 symptoms of illness, inflammation, or soreness.

132

\section{Blood collection}

134 For both Parts A and B, blood was drawn via a 22g needle from a prominent superficial

135 forearm vein located at the antecubital fossa, and drained directly into an $8.5 \mathrm{ml}$ sterile serum

136 separator Vacutainer tube containing a clot activator and gel for serum separation (Beckton

137 and Dickson, USA). Samples were refrigerated at $4^{\circ} \mathrm{C}$ for $30 \mathrm{~min}$ to allow clotting and then

138 centrifuged at $1000 \times g$ at $6^{\circ} \mathrm{C}$ for $10 \mathrm{~min}$ (Rotina 420R, Hettich, Germany). Serum was

139 removed and stored in $400 \mu 1$ aliquots frozen immediately for a maximum of three months at

$140-80^{\circ} \mathrm{C}$ for later analysis. Levels of IL-6 (Quantikine HS600B, R\&D Systems, United States),

141 inducible eHSP72 (HSP72;ADI-EKS-715, Enzo Life Sciences, United States), IgM

142 (AB137982, Abcam PLC, United Kingdom), CRP (hsCRP Immunoassay kit 11190, Oxis

143 International, United States), and LPS (HIT302, Hycult, Biotechnology, Netherlands) were

144 analysed in duplicate by ELISA according to the manufacturer's instructions. The

145 manufacturer stated intra-assay precision was $<10 \%$ for all assays

147 Exercise in the heat protocol (Part B)

148 Participants in Part B undertook an exercise test involving three submaximal workloads of 10

149 min duration $\left(50 \%, 60 \%\right.$ and $\left.70 \% \dot{V} \mathrm{O}_{2 \max }\right)$ on a cycle ergometer followed by a $5 \mathrm{~km}$ time trial

150 (TT) at $35^{\circ} \mathrm{C}$ and $70 \%$ relative humidity (RH) (VeloTron Dynafit Pro and Velotron Coaching 
151 Software, Racermate, United States). Three min rest was given between submaximal

152 workloads and five min rest was given prior to the start of the TT. Participants undertook

153 approximately $40 \mathrm{~min}$ of exercise and were exposed to the hot humid environment for 60-65

154 min. Briefly, the submaximal workloads required the participants to cycle at a fixed wattage

155 between 85-95 rpm. During the TT the participants were able to self-select their gearing and

156 informed of their rpm and distance every 500m. Participants were not aware of their gear,

157 speed, or time elapsed during the TT. A standardised warm-up was undertaken prior to the

$15850 \%$ workload. Heart rate (RS400, Polar Elektro, Finland), and core temperature $\left(\mathrm{T}_{\mathrm{c}}\right)(\mathrm{ttec}$

159 501-3, software version 10.1, Nordex Pty Ltd, Australia; MEAS 449 1RJ rectal temperature

160 thermistor, measurement specialities, Unites States) were sampled at 5 s intervals. Fluid intake

161 (water, ad libitum) and rating of perceived exertion (Borg RPE 6-20) were recorded

162 throughout the test (Borg, 1970). Nude dry body mass was recorded pre and post exercise and

163 fluid loss normalised for body weight and expressed as a percentage change.

164

165 Statistical Analysis

166 The concentration of each biomarker is presented as mean \pm SD. Biomarker reliability was

167 calculated as a coefficient of variation (CV) both within- and -between subjects at day 1 and

168 day 7 and presented as mean $\% \mathrm{CV} \pm 95 \%$ confidence limits (CL). Pre- to post-exercise

169 changes in biomarker concentrations were analysed with paired t-tests and significance was

170 accepted if $\mathrm{p}$ was $<0.05$. Effect sizes for changes in biomarker concentrations were also

171 calculated. The expected reference change, or signal, was estimated for each biomarker as 0.2

$172 \mathrm{x}$ between-subject standard deviation.

173 The criteria to interpret the magnitude of ES were: trivial (0-0.19), small (0.20-0.49),

174 medium (0.50-0.79) and large (0.80 and greater) (Cohen, 1992). The signal to noise ratio

175 score was determined by dividing the reference effect size (signal) by the within-subject test- 
176 retest reliability (noise). The utility of a biomarker was considered 'good' if the expected

177 signal was greater than the noise, or 'unclear' where the signal was less than the noise. A

178 minimum of eight participants was deemed sufficient to detect the smallest worthwhile

179 change between means assuming the reference change was approximately twice the

180 magnitude of the typical error of measurement, with a Type I error of 5\% and Type II error of

$18120 \%$. Biomarker concentrations were log-transformed where appropriate and curve fit was

182 performed using GraphPad Prism Version 6.03 (GraphPad Software Inc, United States)

183 according to the manufacturer instructions. Statistical analyses were performed in IBM SPSS

184 Statistics Version 20 (IBM, United States).

185 Results

186 Part A: Short-term biomarker reliability

187 The biomarker with the lowest within-subject coefficient of variation over the 7 day

188 assessment period (day 1 to day 7) was IL-6 (CV; 19\% $\pm 20 \%$, mean $\pm 95 \%$ CI, ES; 0.16,).

189 CRP had the highest CV $(38 \% \pm 21 \%)$ with a substantially lower level of serum

190 concentration (ES; -0.28) after seven days (Table 1). A comparison of the within-subject

191 variability for each biomarker with an expected reference change is detailed in Table 1.

192 Biomarkers that displayed a good signal to noise ratio were IL-6 and CRP. The expected

193 signal for LPS, IgM and eHSP72 was less than that of the typical noise estimated in this

194 analysis, and therefore the biomarkers were categorised as having unclear or poor reliability

195 (Table 1).

196

TABLE 1 ABOUT HERE

197

Part B: Acute responses of blood biomarkers to exercise in the heat 
198 Blood biomarkers with the largest pre- to post-exercise change were IL-6 and CRP. The

199 blood biomarkers least sensitive to change following the exercise in the heat exposure were

200 IgM, LPS and eHSP72. The exhaustive nature of the exercise task was confirmed with high

201 levels of physiological and perceptual stress: peak $\mathrm{T}_{c} ; 38.9 \pm 0.2{ }^{\circ} \mathrm{C}$, peak heart rate; $187 \pm 5$

202 bpm, reduction in body mass; $1.7 \% \pm 0.3 \%$, and end point RPE; $17 \pm 1$ units. Changes in

203 mean blood biomarker concentration in addition to effect sizes pre-to-post exercise in the

204 heat are presented in Figure 2.

205

FIGURE. 2 ABOUT HERE

206 Discussion

207 The biomarker IL-6 exhibited the smallest within-subject short-term variation (19\%) and the

208 greatest acute pre- to post-exercise change in the heat (4.5 fold change). For the other

209 biomarkers, the short-term resting variation was similar to that of pre- to post-exercise

210 evaluations in the heat, indicating minimal alteration to an acute bout of exercise. It appears

211 only some biomarkers are potentially useful for the purpose of reliably quantifying acute

212 physiological responses in healthy active individuals to hot environmental conditions that

213 elicit modest rises in $\mathrm{T}_{\mathrm{c}}$. Chronic effects on biomarker activity evaluated over a longer period

214 may reveal further insights, such as adaptations to the heat and possible immunosuppression.

215 Even in a resting state, considerable weekly variation was evident for each variable. The

216 cytokine IL-6 exhibited the least within-subject variability of 19\% although other biomarkers

217 such as CRP varied by $38 \%$. The magnitude of this variation is considered concurrently with

218 the expected change in response to an exercise challenge or a period of training, and can be

219 used to inform the decision making process on effects of heat stress (Table 1). Quantifying

220 variation is an inherent part of studying biological systems and can yield important 
221 information when seeking to determine whether or not intervention-induced change in a

222 measured parameter is meaningful.

223 The exercise presented to the participants resulted in a mean core temperature rise of $1.5^{\circ} \mathrm{C}$

224 above baseline levels and the duration of heat exposure was 65 mins, of which 40 mins was

225 dedicated exercise. Although concentrations of IL-6 and the acute phase protein CRP were

226 elevated following exercise, other biomarkers indicative of heat stress such as LPS and

227 eHSP72 did not rise significantly from pre-exposure levels. Serum concentration of IgM also

228 did not rise but instead there was a $15 \%$ reduction in circulation following the exercise bout.

229 It seems plausible that a modest reduction in IgM concentration post exercise reflects the

230 anti-LPS properties of this antibody in response to heat stress. This observation is consistent

231 with the findings of Camus et al. (1998), but not of Hailes et al. (2011) and Lim et al. (2009).

232 The exercise stimulus elicited a response from non-specific pro- and anti-inflammatory blood

233 biomarkers, however it was not sufficient to cause further inflammatory processes associated

234 with heat stress and the inflammatory cascade in healthy, moderately trained males.

235 The significant increase of IL-6 concentration post-exercise may not signify heat stress per

236 se, but rather the stress invoked by the exercise demand itself. IL- 6 can be released into the

237 circulation following various pathological events such as physical exercise, trauma, sepsis,

238 and thermal injury (Moldoveanu, Shephard, and Shek, 2000). There are few studies that have

239 investigated IL-6 as a blood biomarker during exhaustive exercise in the heat, although

240 Selkirk and colleagues (2008) observed a large increase following $2 \mathrm{~h}$ of exhaustive walking

241 in protective clothing in very hot and humid conditions. However, similar effects have been

242 detected following exercise in the absence of a significant heat load. Moldoveanu and

243 colleagues (2000) reported a twenty-fold increase in plasma IL-6 concentrations following $3 \mathrm{~h}$

244 of exercise at $60-65 \%$ of peak oxygen uptake in a thermo-neutral environment - this change is

245 similar in magnitude to that reported by Selkirk et al. (2008). 
246 The large within-subject variation observed for CRP (38\%) raises the question of its

247 suitability as a meaningful biomarker. However, in this study, the biomarker noise (short-

248 term, within-subject variability) was less than that of the signal (response to the exercise task)

249 and there was a medium increase in CRP concentration pre- to post-exercise $(p=0.02, E S$;

250 0.78). Serum levels of CRP can increase rapidly during the acute phase of an inflammatory

251 process (Pepys and Hirschfield, 2003) but this is a non-specific response that could be

252 indicative of infection, illness or other metabolic factors not associated with a heat stimulus.

253 A recent study (Hailes et al., 2011) that measured CRP in serum following 5 consecutive

254 days of exercise in hot and dry conditions $\left(38^{\circ} \mathrm{C}\right.$ and $\left.40 \% \mathrm{RH}\right)$ reported high variability

255 between participants and a standard deviation approximately twice that of the mean after both

256 an acute and ongoing exposure to heat. As the presence of IL-6 is likely to cause an increase

257 in serum levels of CRP (Petersen and Pedersen, 2005), it is likely that the exercise stimulus,

258 and not necessarily the heat load presented to the participants was sufficient to stimulate the

259 release of CRP from the liver. Although both IL-6 and CRP may play important roles in

260 determining the degree of stress placed upon individuals competing or training in more

261 extreme (hot and/or humid) conditions, it seems unlikely this measure would present useful

262 information in terms of responses or adaptations to the heat specifically.

263

264 The low within-subject variability of LPS (CV; 23\%) was encouraging for the practical

265 application of this biomarker for evaluating responses to hot environmental conditions. The

266 low concentrations of LPS observed in this study indicate the participants had the capacity to

267 tolerate the heat load with minimal gut leakage (Pyne et al., 2014). As LPS is the primary

268 endotoxin translocated to circulation under heat load (Yeh et al., 2013), its concentration and

269 regulation is a primary consideration in study of responses to the heat. The outcomes of this

270 study indicate that LPS evaluation in circulating blood should yield reliable results provided 
271 the participants are well rested or are capable of completing a demanding exercise task.

272 Nevertheless, measurement of LPS alone merely indicates the extent of susceptibility to 273 endotoxemia and not the responses of the immune system initiated by this challenge, which

274 can be investigated using other measures such as intestinal fatty acid-binding protein

275 (Morrison, Cheung, and Cotter, 2013), tight junction proteins that indicate increased

276 intestinal permeability (Yeh at al. 2013) or soluble CD14 (Stuempfle, Valentino, Hew-Butler,

277 Hecht, \& Hoffman., 2015). Therefore, to facilitate a comprehensive view of both the

278 underlying endotoxin threat, and compensatory biochemical mechanisms addressing this

279 challenge, it is worthwhile to consider the utility of other viable biomarkers such as IgM and 280 eHSP72.

281

282 The responsiveness of the immune system to release endotoxin is a primary consideration in

283 defence against heat shock. As IgM is a key antibody in neutralising LPS Camus et al., 1998),

284 its concentration in circulating blood can reflect the body's response to endotoxin

285 accumulation, and the likelihood of protective capacity to further challenges. In this study the 286 observed weekly variability of IgM concentration was $28 \%$. The pre- to post-exercise change

287 was $-15 \%$, with 13 of the 20 participants exhibiting a negative change. To our knowledge

288 only one other study has investigated the response of non-specific IgM following exercise in

289 hot and humid conditions (Hailes et al., 2011). However, the reference change reported by

290 Hailes and colleagues (2011) pre- to post-exercise in the heat (CV; 16\%) is smaller than the

291 within-subject variability (noise) reported here (CV; 29\%). It appears that IgM has

292 shortcomings as a viable biomarker for quantifying the anti-LPS response possibly related to

293 the capability of the participants to tolerate the heat load placed upon them, although these

294 data suggest that this response could result in either an increase or decrease in circulating 
295 concentrations. Future research is needed to clarify why some individuals respond in this

296 manner.

297 Inducible eHSP72 exhibited high short-term variability (37\%), however, the pre- to post-

298 exercise change was trivial. In this study the heat load was seemingly not sufficient to induce

299 a significant change in serum concentration of eHSP72. The usefulness of this variable must

300 also be considered against the intended heat load and it may only be useful to quantify the

301 magnitude of response and adaptations to hot environmental conditions, provided the heat

302 stimulus is large enough (Ogura et al., 2008). This may be achieved through longer duration

303 or core temperature clamping protocols and it seems likely that heat loads that cause an

304 increase in core temperature $>39^{\circ} \mathrm{C}$ are needed to evoke LPS translocation and systemic

305 inflammation associated with eHSP72 (Pyne et al., 2014).

306 Between-subject variation also provides useful information for researchers interested in the

307 utility of different measurements. Low within-subject variation indicates that an individual

308 could be expected to provide a similar result on repeated occasions under constant conditions.

309 Therefore, on an individual basis this increases the likelihood that resting or post-exercise

310 measurements could be useful. Conversely, low between-subject variation indicates that all

311 individuals in a cohort exhibit similar concentrations and/or regulate the variable at a similar

312 level. For example, the participants in this study regulated IL-6 at very low and consistent

313 levels. The observation of large between-subject variation for biomarkers such CRP may

314 necessitate the recruitment of more subjects to compress the variation between individuals.

315 However, this type of approach may also limit the interpretation of results and doesn't permit

316 (easy) determination of an individual's response to heat acclimation (Racinais et al., 2013).

317

318 
319 Conclusion

320 Quantifying the inherent variation of biological systems affected by exercise in hot and

321 humid environment informs the choice of inflammatory biomarkers. The utility of the

322 selected biomarkers IL-6 and CRP appears useful even when presented with a high (but

323 tolerable) heat load. However, the short-term variability of other biomarkers such as eHSP72,

324 LPS and IgM overshadows the observed change following 65 mins of exercise and exposure

325 to a hot environment. The within-subject analysis also indicates that individuals consistently

326 regulate the concentration of these biomarkers within homeostatic limits when measured

327 seven days apart. However, the relatively high between-subject variation indicates that it is

328 not possible to establish a standardised concentration of each biomarker suitable for all

329 individuals. Nevertheless, selected biomarkers should be useful for determining the

330 magnitude of responsiveness and stress placed upon individuals training or competing in hot

331 environmental conditions. It appears that a substantial heat stimulus (i.e. $\mathrm{T}_{\mathrm{c}}>39^{\circ} \mathrm{C}$ ) is needed

332 to evoke further responses associated with heat stress and the inflammatory cascade.

333 Conflict of Interest No conflict of interest, financial or otherwise is declared by the 334 authors. 
337

338

339

340

341

342

343

344

345

346

347

348

349

350

351

352

353

354

355

356

357

358

359

\section{References}

Abbasi, A., Fehrenbach, E., Hauth, M., Walter, M., Hudemann, J., Wank, et al. (2013).

Changes in spontaneous and LPS-induced ex vivo cytokine production and mRNA expression in male and female athletes following prolonged exhaustive exercise. Exercise Immunology Review, 19, 8-28.

Ahlers, O., Hildebrandt, B., Dieing, A., Deja, M., Bohnke, T., Wust, P., et al. (2005). Stress induced changes in lymphocyte subpopulations and associated cytokines during whole body hyperthermia of 41.8-42.2 degrees C. European Journal of Applied Physiology, 95(4), 298-306. doi: 10.1007/s00421-005-0009-4.

Borg, G. (1970). Perceived exertion as an indicator of somatic stress. Scandinavian Journal of Rehabilitation Medicine, 2, 92-98.

Camus, G., Nys, M., Poortmans, JR., Venneman, I., Monfils, T., Deby-Dupont, G., et al. (1998). Endotoxaemia, production of tumour necrosis factor alpha and polymorphonuclear neutrophil activation following strenuous exercise in humans. European Journal of Applied Physiology and Occupational Physiology, 79(1), 62-68. DOI: $10.1007 / \mathrm{s} 004210050474$.

Cohen, D., Block, C., Green, M.S,, Lowell, G., and Ofek, I. (1989). Immunoglobulin M, A, and $\mathrm{G}$ antibody response to lipopolysaccharide $\mathrm{O}$ antigen in symptomatic and asymptomatic Shigella infections. Journal of Clinical Microbiology, 27(1), 162-167 .

Cohen, J. (1992). A power primer. Psychological bulletin, 112(1), 155-159.

Garrett, A.T., Rehrer, N.J., and Patterson, M.J. (2011). Induction and decay of short-term heat acclimation in moderately and highly trained athletes. Sports Medicine 41(9), 757-771. doi: 10.2165/11587320-000000000-00000. 
360 Hailes, W.S., Slivka, D., Cuddy, J., and Ruby, B.C. (2011). Human plasma inflammatory

361 response during 5 days of exercise training in the heat. Journal of Thermal Biology, 362 36(5), 277-282. doi: 10.1016/j.jtherbio.2011.03.013.

363 Stuempfle, K. J., Valentino, T., Hew-Butler, T., Hecht, F. M., \& Hoffman, M. D. (2015).

364 Nausea is associated with endotoxemia during a 161-km ultramarathon. Journal of

$365 \quad$ Sports Sciences, 1-7. DOI: 10.1080/02640414.2015.1130238.

366 Lim, C.L., Pyne, D.B., Horn, P., Kalz, A., Saunders, P., Peake, J., et al. (2009). The effects of

367 increased endurance training load on biomarkers of heat intolerance during intense

368 exercise in the heat. Applied Physiology, Nutrition, and Metabolism, 34(4), 616-624.

369 doi: $10.1139 / \mathrm{H} 09-021$.

370 Marshall, H.C., Campbell, S.A., Roberts, C.W., and Nimmo, M.A. (2007). Human

371 physiological and heat shock protein 72 adaptations during the initial phase of humid-

372 heat acclimation. Journal of Thermal Biology, 32, 341-348. doi:

$373 \quad 10.1016 /$ j.jtherbio.2007.04.003

374 Mestre-Alfaro, A., Ferrer, M.D., Banquells, M., Riera, J., Drobnic, F., Sureda, A., et al.

375 (2012). Body temperature modulates the antioxidant and acute immune responses to

$376 \quad$ exercise. Free Radical Research, 46(6), 799-808. doi:

$377 \quad 10.3109 / 10715762.2012 .680193$.

378 Moldoveanu, A.I, Shephard, R.J., and Shek, P.N. (2000). Exercise elevates plasma levels but

379 not gene expression of IL-1 $\beta$, IL-6, and TNF- $\alpha$ in blood mononuclear cells. Journal of

380 Applied Physiology, 89(4), 1499-1504. PMID: 11007588.

381 Morrison, S.A., Cheung, S.S., and Cotter, J.D. (2014). Bovine colostrum, training status, and

382 gastrointestinal permeability during exercise in the heat: a placebo controlled double

383 blind study. Applied Physiology, Nutrition, and Metabolism, 39, 1070-1082. doi:

$384 \quad$ 10.1139/apnm-2013-0583. 
385 Natelson, B.H., Zhou, X., Ottenweller, J.E., Bergen, M.T., Sisto, S.A., Drastal, S., et al.

386 (1996). Effect of acute exhausting exercise on cytokine gene expression in men.

387 International Journal of Sports Medicine, 17, 299-302. PMID: 8814513.

388 Ogura, Y., Naito, H., Akin, S., Ichinoseki-Sekine, N., Kurosaka, M., Kakigi, R., et al. (2008).

389 Elevation of body temperature is an essential factor for exercise-increased

390 extracellular heat shock protein 72 level in rat plasma. American Journal of

391 Physiology. Regulatory, Integrative and Comparative Physiology, 294(5), R1600-

$392 \quad$ R1607. doi: 10.1152/ajpregu.00581.2007.

393 Pepys, M.B., and Hirschfield, G.M. (2003). C-reactive protein: a critical update. Journal of

394 Clinical Investigation, 111(12), 1805-1812. doi:10.1172/JCI18921.

395 Petersen, A.M., and Pedersen, B.K. (2005). The anti-inflammatory effect of exercise. Journal

396 of Appled Phsyiology, 98(4), 1154-1162. doi: 10.1152/japplphysiol.00164.2004.

397 Pilch, W., Szygula, Z., Tyka, A.K, Palka, T., Tyka, A., Cison, T., et al. (2014). Disturbances

398 in Pro-Oxidant-Antioxidant Balance after Passive Body Overheating and after

399 Exercise in Elevated Ambient Temperatures in Athletes and Untrained Men. PloS

$400 \quad$ one, 9(1), e85320. doi: 10.1371/journal.pone.0085320.

401 Pyne, D.B., Guy, J.H., and Edwards, A.M. (2014). Managing Heat and Immune Stress in

402 Athletes With Evidence-Based Strategies. International Journal of Sports Physiology

403 and Performance, 9(5), 744-750 doi: 10.1123/ijspp.2014-0232.

404 Racinais, S., Mohr, M., Buchheit, M., Voss, S.C., Gaoua, N., Grantham, J., et al. (2013).

405 Individual responses to short-term heat acclimatisation as predictors of football

406 performance in a hot, dry environment. British Journal of Sports Medicine, 46(11),

407 810-815. doi: 10.1136/bjsports-2012-091227.

408 Rhind, S.G., Gannon, G.A., Shephard, R.J., Buguet, A., Shek, P.N., and Radomski, M.W.

409 (2004). Cytokine induction during exertional hyperthermia is abolished by core 
410 temperature clamping: neuroendocrine regulatory mechanisms. International Journal

411 of Hyperthermia, 20(5), 503-516. doi: 10.1080/02656730410001670651.

412 Scheele, C., Nielsen, S., and Pedersen, B.K. (2009). ROS and myokines promote muscle 413 adaptation to exercise. Trends in Endocrinology and Metabolism, 20(3), 95-99. doi:

$414 \quad 10.1016 /$ j.tem.2008.12.002.

415 Selkirk, G.A., McLellan, T.M., Wright, H.E., and Rhind, S.G. (2008). Mild endotoxemia,

$416 \mathrm{NF}-\kappa \mathrm{B}$ translocation, and cytokine increase during exertional heat stress in trained and

417 untrained individuals. American Journal of Physiology. Regulatory, Integrative and

418 Comparative Physiology, 295(2), R611-R623. doi: 10.1152/ajpregu.00917.2007.

419 Singh, L.P., Kapoor, M., and Singh, S.B. (2013). Heat: not black, not white. It's gray!!!

$420 \quad$ Journal of Basic and Clinical Physiology and Pharmacology, 24(4), 209-224. doi:

$421 \quad 10.1515 / \mathrm{jbcpp}-2012-0080$.

422 Wright, H.E., McLellan, T.M., Larose, J., Hardcastle, S.G., Boulay, P., and Kenny, G.P.

423 (2013). Are circulating cytokine responses to exercise in the heat augmented in older

424 men? Applied Physiology, Nutrition, and Metabolism, 39, 117-123. doi:

$425 \quad 10.1139 /$ apnm-2013-0223.

426 Yeh, Y.J., Law, L.Y., and Lim, C.L. (2013). Gastrointestinal response and endotoxemia 427 during intense exercise in hot and cool environments. European Journal of Applied 428 Phyisology, 113(6), 575-1583. doi: 10.1007/s00421-013-2587-x. 
Table 1. Coefficient of variation both within (day zero to day seven) and between subjects with inferences to the reliability and usefulness of selected biomarkers

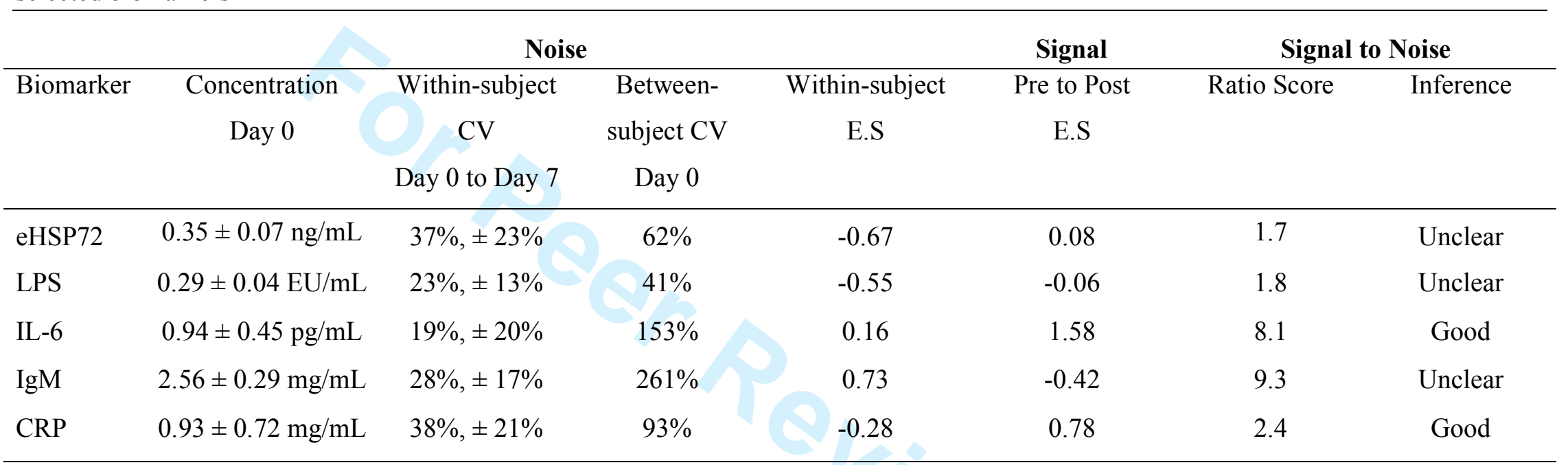

Biomarker concentrations are presented as mean \pm SD, within-subject coefficient of variation (CV) is presented as mean, $\pm 95 \%$ CI. E.S; Effect size (Cohen's d). Withinsubject effect size was calculated from the typical change in the mean (raw units) of the measured parameter from day 0 to day 7; reference effect size was calculated from the typical change (pre \& post mean \& SD) in the measured parameter from selected intervention studies. Ratio score was calculated by dividing the reference effect size by the within-subject effect size. CRP; C-reactive protein. eHSP72; extracellular heat shock protein. IL-6; interleukin-6. LPS; lipopolysaccharide. IgM;

immunoglobulin $\mathrm{M}$ 


\section{Figure Captions.}

432

433 Figure 1. Schematic illustration of the experimental procedures showing that blood was 434 sampled at D 1 (day one, Part A and Part B) and D 7 (Day seven, Part A). ExH; Exercise in 435 the heat intervention (Part B).

436

437 Figure 2. Serum biomarker concentrations presented as mean \pm SD from Part A (Short-term; 438 Day 1 and Day 7) and Part B (Exercise in the heat; Pre and Post). * = significantly different 439 from pre concentration. CRP; C-reactive protein. eHSP72; extracellular heat shock protein. 440 IL-6; interleukin-6. LPS; lipopolysaccharide. IgM; immunoglobulin M 
Figure 1. Schematic illustration of the experimental procedures showing that blood was sampled at $D 1$ (day one, Part A and Part B) and D 7 (Day seven, Part A). ExH; Exercise in the heat intervention (Part B).

\section{$254 \times 190 \mathrm{~mm}(300 \times 300 \mathrm{DPI})$}



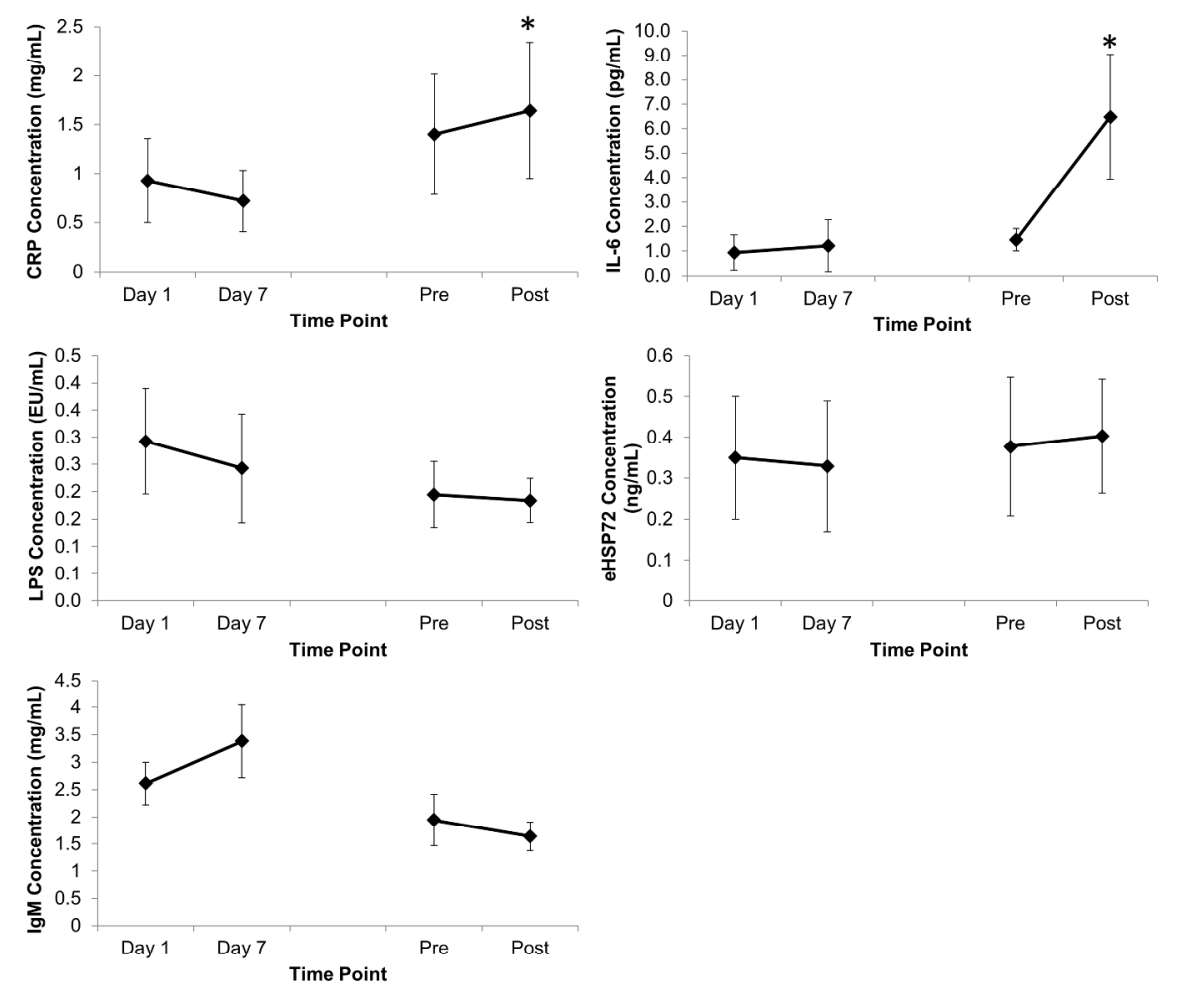

Figure 2. Serum biomarker concentrations presented as mean \pm SD from Part A (Short-term; Day 1 and Day 7) and Part B (Exercise in the heat; Pre and Post). * = significantly different from pre concentration. CRP; C-reactive protein. eHSP72; extracellular heat shock protein. IL-6; interleukin-6. LPS; lipopolysaccharide. IgM; immunoglobulin M $287 \times 309 \mathrm{~mm}(252 \times 252 \mathrm{DPI})$ 